OPEN ACCESS

Edited by:

László Kopper,

Semme/weis University, Hungary

*Correspondence:

Shengjun Xiao

xiaoshengjun@glmc.edu.cn

Jiaxue WeI

weijiaxue@hotmail.com

"These authors have contributed equally to this work

+ORCID:

Huoying Chen,

https://orcid.org/0000-0003-0796-

5975 ;

Xiaoying Lin,

https://orcid.org/0000-0003-0376-

$712 X ;$

Shengjun Xiao,

https://orcid.org/0000-0001-6731-

1899

Received: 21 September 2020 Accepted: 26 February 2021

Published: 31 March 2021

Citation:

Chen H, Lin X, Liu H, Huang C, Li R, Ai J, Wei J and Xiao S (2021) HMGB1

Translocation is Associated with

Tumor-Associated Myeloid Cells and Involved in the Progression of

Fibroblastic Sarcoma.

Pathol. Oncol. Res. 27:608582.

doi: 10.3389/pore.2021.608582

\section{HMGB1 Translocation is Associated with Tumor-Associated Myeloid Cells and Involved in the Progression of Fibroblastic Sarcoma}

\author{
Huoying Chen ${ }^{1,2 \dagger \#, ~ X i a o y i n g ~ L i n ~}{ }^{1+\#, ~ H o n g b o ~ L i u ~}{ }^{2}$, Cheng Huang ${ }^{1}$, Rong $\mathrm{Li}^{1}$, Jie $\mathrm{Ai}^{2}$, \\ Jiaxue $\mathrm{Wei}^{1 *}$ and Shengjun Xiao ${ }^{3+*}$ \\ ${ }^{1}$ Prenatal Diagnosis Center, Guangdong Second Provincial General Hospital, Guangdong Provincial Emergency Hospital, \\ Guangzhou, China, ${ }^{2}$ Department of Laboratory Medicine, The Second Affiliated Hospital of Guilin Medical University, Guilin, \\ China, ${ }^{3}$ Department of Pathology, The Second Affiliated Hospital of Guilin Medical University, Guilin, China
}

The morphological variability and genetic complexity of fibroblastic sarcoma makes its diagnosis and treatment a challenge. High-mobility group box 1 protein (HMGB1), which functions as a DNA chaperone and a prototypical damage-associated molecular pattern, plays a paradoxical role in cancer. However, the expression pattern and role of HMGB1 in fibroblastic sarcomas is ill defined. By immunostaining of 95 tissue microarray cores of fibroblastic sarcomas, HMGB1 was found to be expressed in most tumor tissues. Nuclear HMGB1 translocation to cytoplasm was observed both in tumor cells and vascular endothelial cells. A visible number of tumor-associated myeloid cells including CD68 ${ }^{+}$ and $\mathrm{CD}_{163^{+}}$macrophages and $\mathrm{CD}_{3} 3^{+}$myeloid cells were also detected in most tumor tissues. HMGB1 translocation was not only associated with CD68, CD163, and CD33 density, but also with disease progression. These results imply that HMGB1, an important regulator of the tumor microenvironment, is associated with tumor-associated myeloid cells and involved in the progression of fibroblastic sarcomas; HMGB1 may serve as a promising prognostic biomarker and a potential therapeutic target for fibroblastic sarcoma.

Keywords: high-mobility group box 1 protein, translocation, sarcoma, tumor-associated macrophage, myeloid cell

\section{INTRODUCTION}

Fibroblastic sarcoma is a common panel of soft tissue sarcoma that accounts for approximately $14 \%$ of all sarcomas [1] and $12 \%$ of pediatric soft tissue tumors [2]. Due to morphologic variability and genetic complexity, the diagnosis and treatment of fibroblastic sarcoma remains a challenge. According to the new 2013 World Health Organization classification, intermediate and malignant fibroblastic sarcoma includes dermatofibrosarcoma protuberans (DFSP), adult-type fibrosarcoma (ATFS), myxofibrosarcoma (MFS) and other histologic subtypes with recurrent cytogenetic or molecular genetic abnormalities [3]. At present, like other sarcomas, the first-line treatment strategy of fibroblastic sarcoma is mainly the combination of surgery, chemotherapy, radiotherapy and other systemic treatment [4]. However, there are limited effective therapy options for treatment as failure often results from local recurrence and distant metastasis [5]. Although new chemotherapeutic drugs such as aldoxorubicin [6], amrubicin [7] and eribulin [8], as well as immune checkpoint blockade agents such as ipilimumab [9] and pembrolizumab [10] for advanced sarcoma are evolving, their approvals are limited to some select histologic subtypes with improved outcomes. 
Therefore, studies on exploring novel biomarkers involved in the progression of fibroblastic sarcoma and their potential as therapeutic targets are still necessary.

High-mobility group box 1 protein (HMGB1), which performs dual functions as a highly conserved chromosomal protein that enhances transcription and is a crucial cytokine that mediates the response to infection, injury and inflammation, has been reported to play a paradoxical role in cancer $[11,12]$. During tumor development and in cancer therapy, extracellular HMGB1 can not only contribute to tumorigenesis but also can stimulate anti-tumor immune responses thought binding to various receptors on different types of cells, such as receptor for advanced glycation end products (RAGE), toll-like receptors (TLRs), chemokine (C-X-C motif) receptor 4 (CXCR4), and $\mathrm{T}$ cell immunoglobulin mucin 3 (TIM3) [12-15]. It has been reported that HMGB1 promotes human embryonic lung fibroblast proliferation and extracellular matrix production [16], induces synovial fibroblasts angiogenesis via hypoxiainducible factor (HIF)-1 $\alpha$ activation [17], and induces cardiac fibroblasts migration via CXCR4 in a chemokine $(\mathrm{C}-\mathrm{X}-\mathrm{C}$ motif) ligand 12 (CXCL12) -independent manner [18], which implies that HMGB1 may be involved in the pathogenesis and progression of fibroblastic tumors.

There have been several studies on the role of HMGB1 in fibrosarcoma cells in vitro. HMGB1-RAGE signals have been reported to exacerbate the malignant phenotype of HT1080 human fibrosarcoma cell lines [19]. During chemotherapy or radiotherapy, HMGB1 released by dying MCA205 fibrosarcoma cells activated tumor antigen-specific T-cell immunity through acting on TLR4 expressed by antigen presenting cells [20]. Tumor-associated myeloid cells represented by tumorassociated macrophages (TAMs) and myeloid-derived suppressor cells (MDSCs) express multiple receptors of HMGB1 and play an important role in supporting cancer growth and survival, angiogenesis, metastasis as well as immunosuppression [21]. The action of HMGB1 on tumorassociated myeloid cells and their impact on the progression of fibroblastic sarcoma naturally attract attention.

However, no additional studies have focused on the expression and possible role of HMGB1 in fibroblastic sarcomas. Therefore, this study aimed to explore the expression pattern and role of HMGB1 in fibroblastic sarcomas, as well as its relationship with tumor-associated myeloid cells, hoping to provide clues as to whether HMGB1 can be a potential therapeutic target for the disease.

Immunostaining used in this study showed that abundant HMGB1 was expressed in most tumor tissues. Cytoplasmstaining revealed that HMGB1 was present both in tumor cells and vascular endothelial cells. A visible group of TAMs and CD33-positive myeloid cells were also observed in most tumor tissues. HMGB1 expression was not only related to disease progression, but also closely related to tumor-associated myeloid cells. This study implies that HMGB1 plays an important role in the progression of fibroblastic sarcoma and may serve as a useful prognostic biomarker and a potential therapeutic target for the disease.
TABLE 1 | Baseline patient characteristics.

\begin{tabular}{|c|c|}
\hline Characteristic & $N(\%)$ (Total $N=95)$ \\
\hline \multicolumn{2}{|l|}{ Age } \\
\hline$<55$ & 77 (81.05) \\
\hline$\geq 55$ & 18 (18.95) \\
\hline \multicolumn{2}{|l|}{ Sex } \\
\hline Female & 39 (41.05) \\
\hline Male & 56 (58.95) \\
\hline \multicolumn{2}{|l|}{ Tumor site } \\
\hline Skin & $54(56.84)$ \\
\hline Extremity & $23(24.21)$ \\
\hline Trunk and neck & 18 (18.95) \\
\hline \multicolumn{2}{|l|}{ Histologic diagnosis } \\
\hline DFSP & $54(56.84)$ \\
\hline MFS & $12(12.63)$ \\
\hline ATFS & $29(30.53)$ \\
\hline \multicolumn{2}{|l|}{ TNM staging } \\
\hline T1aNOMO+T1bNOMO & 63 (66.32) \\
\hline T2aNOMO+T2bNOMO & 32 (33.68) \\
\hline \multicolumn{2}{|l|}{ Tumor grade } \\
\hline G1 & $55(57.89)$ \\
\hline G2 & 35 (36.84) \\
\hline G3 & $5(5.26)$ \\
\hline \multicolumn{2}{|l|}{ AJCC staging } \\
\hline$I A+I B$ & $55(57.89)$ \\
\hline$\|A+\| B$ & $38(40.00)$ \\
\hline III & $2(2.11)$ \\
\hline
\end{tabular}

Abbreviations: N, Number; TNM, Tumor Node Metastasis; AJCC, American Joint Committee on Cancer; DFSP, Dermatofibrosarcoma protuberans; MFS, Myxofibrosarcoma; ATFS, Adult-type fibrosarcoma.

\section{MATERIALS AND METHODS}

\section{Ethics Approval}

The process of case collection was approved by Ethics Committee of People's Hospital of Tongxu County (Henan Province, China). Informed consent from patients was not necessary because the specimen were analyzed retrospectively and already belonged to National Human Genetic Resources Sharing Service Platform (Library number: 2005DKA21300) at the time of the analyses.

\section{Tissue Microarray}

Fibroblastic sarcomas used for construction of tissue microarray (TMA) in this study were obtained from the National Human Genetic Resources Sharing Service Platform (Library number: 2005DKA21300). Ninety-five formalin-fixed, paraffin-embedded tissue blocks of fibroblastic sarcomas including DFSP, MFS and ATFS were selected for construction of the TMA, which was generated by Alenabio (Xi'an, China). Hematoxylin and eosin-stained slides from each tissue block were read by a senior consultant pathologist to obtain $1.0 \mathrm{~mm}$-diameter core biopsies of primary fibroblastic sarcomas. The patient characteristics were also collected and summarized in Table 1. However, follow-up information from the patients was unavailable.

\section{Immunohistochemistry}

Immunohistochemistry (IHC) was performed on TMA slides with the anti-HMGB1 (1:2000; Abcam, Cambridge, MA, 
United States), anti-Cluster of Differentiation (CD) 163 (1:200; Abcam, Cambridge, MA, United States), anti-CD33 (1:50; R\&D Systems, Minneapolis, MN, United States), and anti-CD68 (Ready-to-use antibody, MXB Biotechnologies, Fuzhou, Fujian Province, China) antibodies using a MaxvisionTM2 HRPPolymer anti-Mouse/Rabbit IHC Kit according to the manufacturer's instructions (MXB Biotechnologies, Fuzhou, Fujian Province, China). All slides were counterstained with hematoxylin and mounted. Digital images of immunostained TMA slides were acquired using the KF-PRO-005-EX digital pathology slide scanner (KFBIO, Ningbo, Zhejiang Province, China).

\section{Histological Scoring}

The immunostaining score of HMGB1 was divided into total score, nucleus-staining score and cytoplasm-staining score. The nucleus-staining and cytoplasm-staining of HMGB1 were scored on a scale semiquantitatively based on the percentage of positive cells and staining intensity by the following method. Six fields per TMA core at $\times 400$ magnification were randomly selected. The percentages of HMGB1-positive cells with nucleus-staining or cytoplasm-staining were calculated and scored as follows: 1) score $0,<5 \% ; 2$ ) score $1, \geq 5 \%$ and $<25 \% ; 3$ ) score $2, \geq 25 \%$ and $<50 \% ; 4$ ) score $3, \geq 50 \%$. And the staining intensity of HMGB1-positive cells with nucleus-staining or cytoplasm-staining were evaluated and scored as follows: 1 ) score 0 , negative; 2 ) score 1 , weak; 3 ) score 2, moderate; 4) score 3 , strong. The nucleus-staining or cytoplasm-staining score of one field was the sum of the percentage of positive cells and the staining intensity scores. The final score of each TMA core was the mean of the six fields, and the total score of HMGB1 was the sum of nucleusstaining and cytoplasm-staining scores. Scoring was performed by a pathologist experienced in scoring tumor biomarkers. And then statistical analysis was performed on HMGB1 total score, nuclear staining score and cytoplasmic staining score separately.

Immunohistochemical markers of immune cells, including CD68, CD163 and CD33, were scored by counting the number of positive-staining cells per TMA core divided by the area of the core to yield a value for cells $/ \mathrm{mm}^{2}$. Cell counting was performed by two investigators using the Image-Pro Plus 6.0 software (Media Cybernetics, Rockville, MD, United States). Scoring was calculated from the mean of the two independently conducted assessments.

\section{Statistics}

Statistical analyses were performed using SPSS standard version 16.0 (SPSS Inc. Chicago, IL) and GraphPad Prism version 5.0 (GraphPad Software, SanDiego, CA). Comparisons between groups were analyzed by independent sample $t$-test, one-way analysis of variance (ANOVA) followed by Bonferroni's test or by Dunnett's T3 test (for non-normal data with unequal variances) for quantitative data, or Mann-Whitney $U$ test for ordinal data, as appropriate. The correlation between histological score, cell density and tumor grade was determined by Spearman rank test. $p$-values $<0.05$ were considered statistically significant.

\section{RESULTS}

\section{The Immunostaining Pattern of HMGB1 in Fibroblastic Sarcomas}

In order to clarify the expression pattern of HMGB1 in fibroblastic sarcomas, the staining of HMGB1 were detected by IHC assay in 95 TMA cores of fibroblastic sarcomas, which included 54 cases of DFSP, 12 cases of MFS and 29 cases of ATFS. The total score, nucleus-staining score and cytoplasm-staining score of HMGB1 per TMA core were then evaluated and statistically analyzed.

Under physiological conditions, HMGB1 is usually located in the nucleus. During tumor development and in cancer therapy, HMGB1 is released and plays multiple roles through binding receptors, including RAGE, TRL2/4, TIM3 and CXCR4 [12]. Before releasing, HMGB1 must first be translocated from the nucleus to the cytoplasm [22]. In this study, as shown in Table 2, there was no difference in HMGB1 nucleus-staining score in TMA cores with different tumor staging and grades. However, TMA cores with higher Tumor Node Metastasis (TNM) staging and American Joint Committee on Cancer (AJCC) staging, as well as higher tumor grades, had higher HMGB1 cytoplasm-staining score and HMGB1 total score $(p<0.05)$. These results indicated that the difference in HMGB1 expression in tumors with different staging and grades was mainly the difference in HMGB1 cytoplasmic expression. Representative images in Figures 1A,B showed the expression and cytoplasm-staining of HMGB1 in TMA cores with different grades. Cytoplasm-staining of HMGB1 in tumor cells was easily observed in most of the TMA cores. The higher the tumor grade, the easier to observe the cytoplasm staining of HMGB1 in more tumor cells (Figure 1B). Moreover, correlation analysis revealed that HMGB1 cytoplasm-staining score and HMGB1 total score were related to tumor grade $(p=0.0049, r=0.2863$; $p=$ $0.0005, \quad r=0.3508$; respectively) (Figure 1C), whereas HMGB1 nucleus-staining score was not related (data not shown). Cytoplasm-staining of HMGB1 in vascular endothelial cells was also observed in some of the TMA cores (Figure 1D). This means that, in addition to tumor cells, HMGB1 could also be released from vascular endothelial cells to play a certain role in fibroblastic sarcomas. These results suggest that HMGB1 derived from tumor cells and vascular endothelial cells may be involved in the progression of fibroblastic sarcomas.

\section{Immunostaining of CD68, CD163 and CD33 in Fibroblastic Sarcomas}

In order to clarify the accumulation of TAMs and MDSCs in fibroblastic sarcomas, the desity of CD68 (a pan-macrophage marker), CD163 (a M2-polarized macrophage marker) and CD33 (a myeloid marker that sometimes is used for identification of MDSCs [23]) in 95 TMA cores of fibroblastic sarcomas were also evaluated by immunostaining. As shown in Table 3 and Figure 2A, a considerable number of CD68 [median (range): $243(13-3,753)$ cells $/ \mathrm{mm}^{2}$ ] and CD163-positive [median (range): $245(11-4,517)$ cells $\left./ \mathrm{mm}^{2}\right]$ cells were observed in most of the 
TABLE 2 | Relationship between HMGB1 score and patient characteristics of fibroblastic sarcomas.

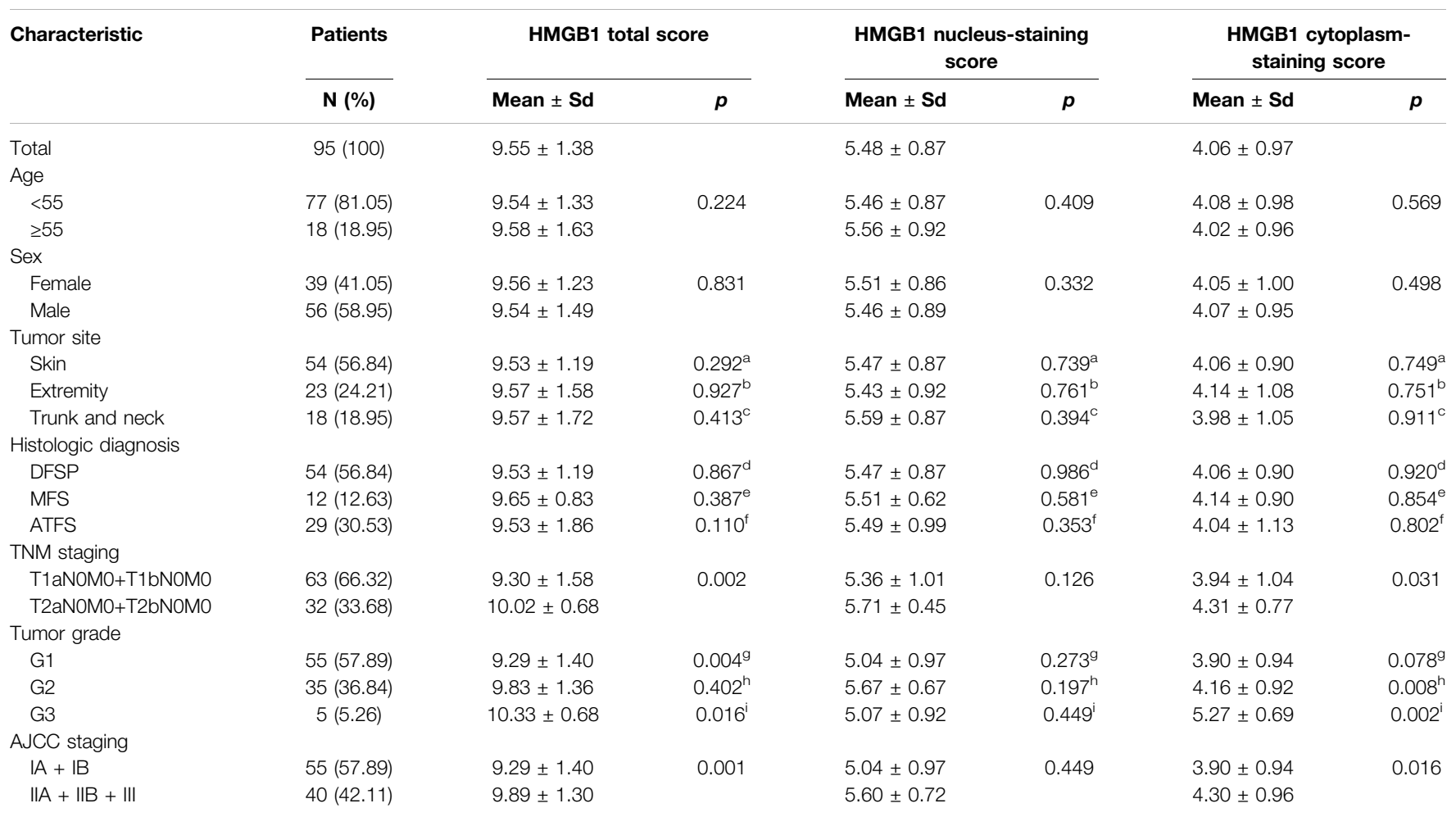

Note: a, Skin group vs Extremity group; $b$, Extremity group vs Trunk and neck group; c, Trunk and neck group vs Skin group; $d$, DFSP group vs MFS group; e, MFS group vs ATFS group; $f$, ATFS group vs DFSP group; g, G1 vs G2; $h$, G2 vs G3; i, G3 vs G1; using Mann-Whitney U test.

Abbreviations: N, Number; TNM, Tumor Node Metastasis; AJCC, American Joint Committee on Cancer; DFSP, Dermatofibrosarcoma protuberans; MFS, Myxofibrosarcoma; ATFS, Adult-type fibrosarcoma; HMGB1, high-mobility group box 1.

TMA cores. Moreover, TMA cores with higher AJCC staging had higher density of CD163 but not CD68 $(p<0.05)$. Table 3 and Figure 2A also showed that a small group of CD33-positive cells [median (range): $17(0-3,078)$ cells $/ \mathrm{mm}^{2}$ ] were observed in most of the TMA cores. Although there was a tendency toward a higher CD33 density in tumors with higher TNM staging and AJCC staging, this did not reach statistical significance (Table 3). These results suggest that tumor-associated myeloid cells including TAMs and CD33-positive myeloid cells may play an important role in promoting the development of fibroblastic sarcoma.

\section{Expression Correlation of HMGB1 and CD68, CD163 and CD33 in Fibroblastic Sarcomas}

The study explored the relationship between HMGB1 and the density of tumor-associated myeloid cells in fibroblastic sarcomas. Representative images of high, medium or low staining of HMGB1, CD68, CD163 and CD33 in one same TMA core were shown in Figure 2A. Correlation analysis revealed that the density of CD68, CD163 and CD33 was not only positively correlated with the total score of HMGB1, but also with the cytoplasm-staining score of HMGB1 in fibroblastic sarcomas (Figure 2B). However, there was no correlation between HMGB1 nucleus-staining score and CD68, CD163 and CD33 density (data not shown). Taken together, these results indicate that $\mathrm{HMGB} 1$ may be released and act on CD68 and CD163-positive M2-polarized macrophages and CD33-positive myeloid cells in fibroblastic sarcomas, and involved in disease progression.

\section{DISCUSSION}

HMGB1 plays a significant role in many cancers and has promising clinical application prospects as a therapeutic target. Several HMGB1-targeting agents have been developed and used in experimental cancer research. These agents include sRAGE [24], HMGB1 neutralizing antibody [25], A box protein [25], ethyl pyruvate [26], quercetin and glycyrrhizin [27], and platinating agents such as cisplatin and oxaliplatin [28]. However, the relationship between HMGB1 and soft tissue sarcoma has received little attention.

This study detected the expression pattern of HMGB1 in several types of fibroblastic sarcomas including DFSP, MFS and ATFS by immunostaining. HMGB1 was found to be expressed in most tumor cells, and a higher expression of HMGB1 was observed in tumor tissues with higher grade and staging. The correlation between the high expression of HMGB1 and tumor grade and staging has also been reported in gastric adenocarcinomas [29] and liver cancer [30]. Furthermore, 


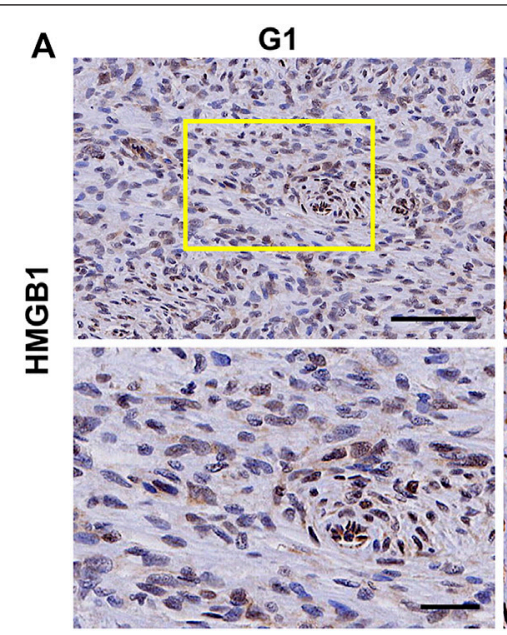

B

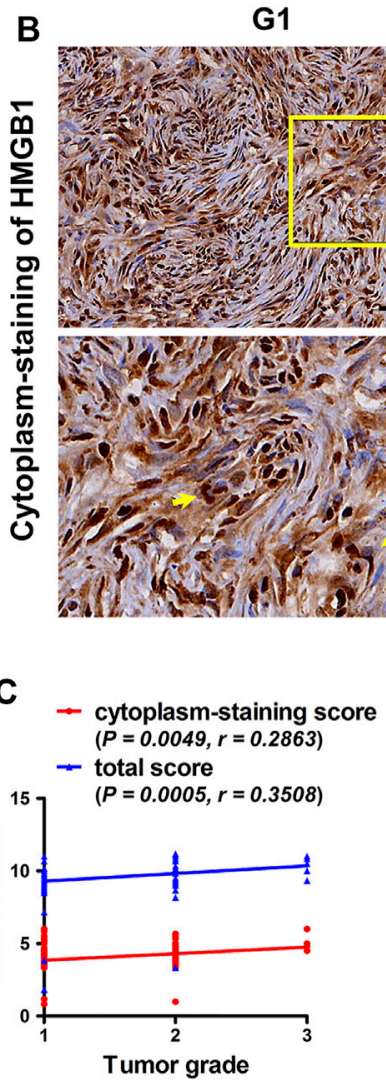

\section{D}

G2

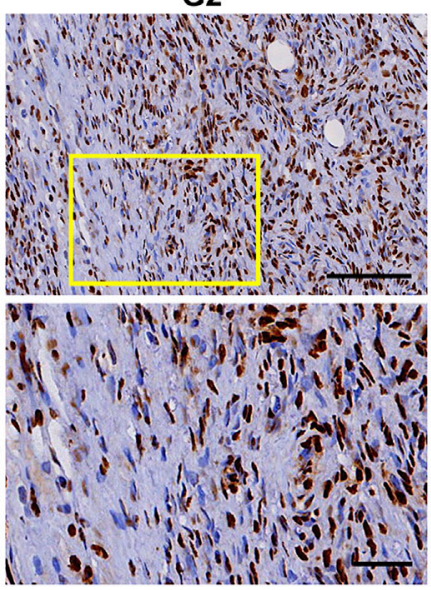

G2

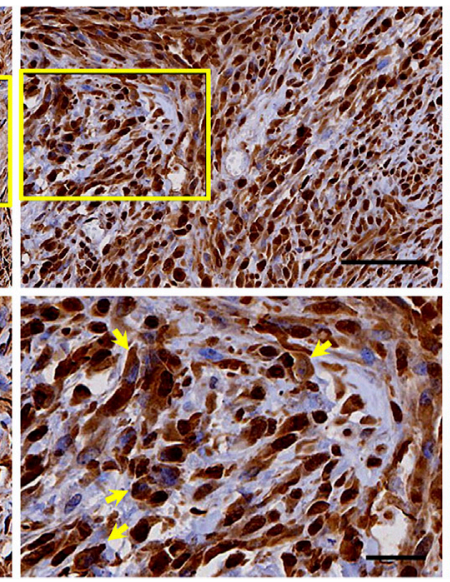

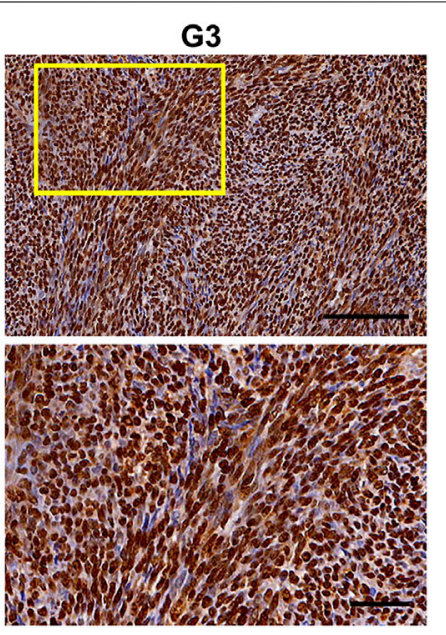

G3

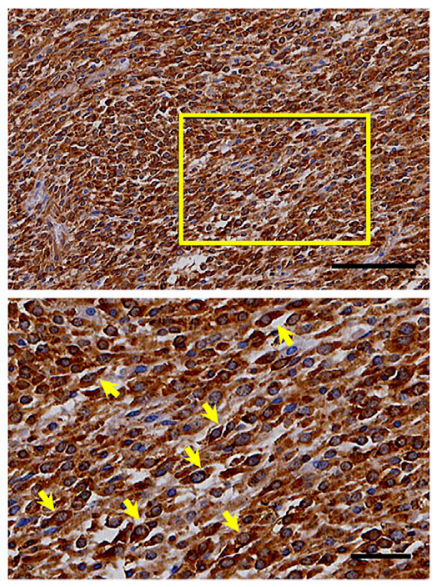

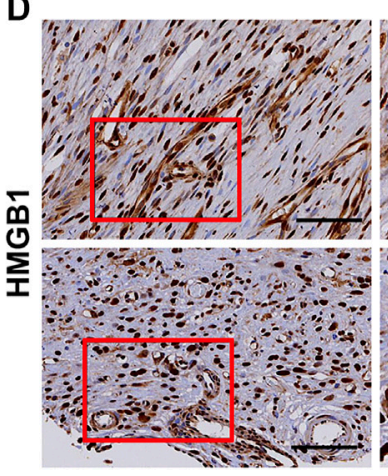
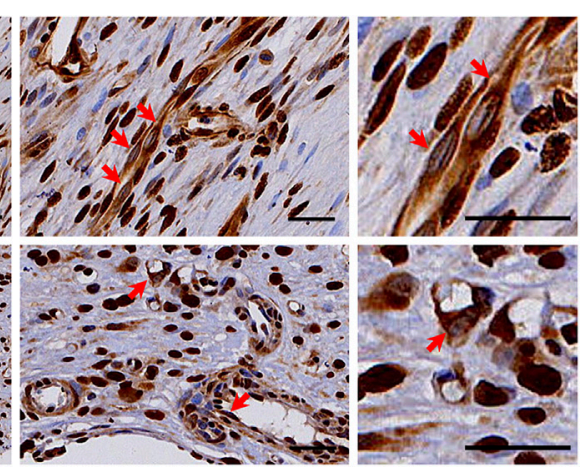

FIGURE 1 | The expression pattern of HMGB1 in fibroblastic sarcomas. (A) Representative images of immunostaining of HMGB1 in tumors with different tumor grade. (B) Representative images of cytoplasm-staining of HMGB1 in tumors with different tumor grade. HMGB1 cytoplasm-staining-positive tumor cells are indicated by yellow arrow at high magnification, which is framed by yellow rectangles at low magnification. (C) Correlation of HMGB1 total score and cytoplasm-staining score with tumor grade in fibroblastic sarcomas. The correlation was determined by Spearman rank correlation test. (D) Cytoplasm-staining of HMGB1 in vascular endothelial cells. Cytoplasm-staining HMGB1-positive vascular endothelial cells are indicated by red arrow at high magnification, which is framed by red rectangles at low magnification. Scale bars were $100 \mu \mathrm{m}$ for low magnification $(\times 200)$ and $50 \mu \mathrm{m}$ for high magnification $(\times 400)$ and for the highest magnification in $(\mathbf{D})$.

obvious cytoplasm-staining of HMGB1 was observed both in tumor cells and vascular endothelial cells and positively correlated with tumor grade in fibroblastic sarcoma. Consistently, cytoplasmic expression of HMGB1 was also detected in breast cancer and human renal clear cell cancer and indicated higher tumor grades [31-33]. These results suggest that HMGB1 could be released by tumor cells and vascular endothelial cells and involved in the progression of fibroblastic sarcoma. 
TABLE 3 | Relationship between CD68, CD163 and CD33 density and patient characteristics of fibroblastic sarcomas.

\begin{tabular}{|c|c|c|c|c|c|c|c|}
\hline \multirow[t]{2}{*}{ Characteristic } & \multirow{2}{*}{$\frac{\text { Patients }}{\mathrm{N}(\%)}$} & \multicolumn{2}{|c|}{$\mathrm{CD} 8^{+}$cells $/ \mathrm{mm}^{2}$} & \multicolumn{2}{|c|}{$\mathrm{CD}_{163^{+}}$cells $/ \mathrm{mm}^{2}$} & \multicolumn{2}{|c|}{ CD33 $^{+}$cells $/ \mathrm{mm}^{2}$} \\
\hline & & Median (range) & $p$ & Median (range) & $p$ & Median (range) & $p$ \\
\hline Total & $95(100)$ & $243(13-3,753)$ & & $245(11-4,517)$ & & $17(0-3,078)$ & \\
\hline \multicolumn{8}{|l|}{ Age } \\
\hline$<55$ & 77 (81.05) & $220(13-3,753)$ & 0.648 & $231(11-4,517)$ & 0.833 & $14(0-3,078)$ & 0.519 \\
\hline$\geq 55$ & 18 (18.95) & 302.5 (33-627) & & $276.5(84-1,899)$ & & $49.5(3-161)$ & \\
\hline \multicolumn{8}{|l|}{ Sex } \\
\hline Female & $39(41.05)$ & $254(17-2,419)$ & 0.593 & $245(11-4,487)$ & 0.344 & $14(0-1,582)$ & 0.277 \\
\hline Male & 56 (58.95) & $223.5(13-3,753)$ & & $245(17-4,517)$ & & $18(0-3,078)$ & \\
\hline \multicolumn{8}{|l|}{ Tumor site } \\
\hline Skin & $54(56.84)$ & $297.5(45-2,451)$ & $1.000^{\mathrm{a}}$ & $260(17-4,260)$ & $1.000^{\mathrm{a}}$ & $11(0-3,078)$ & $1.000^{\mathrm{a}}$ \\
\hline Extremity & $23(24.21)$ & $93(22-3,753)$ & $1.000^{\mathrm{b}}$ & $232(28-4,517)$ & $1.000^{\mathrm{b}}$ & $20(0-1,997)$ & $1.000^{b}$ \\
\hline Trunk and neck & 18 (18.95) & $138.5(13-2,419)$ & $1.000^{\mathrm{C}}$ & $185.5(11-4,487)$ & $1.000^{\mathrm{C}}$ & $23.5(0-1,582)$ & $1.000^{\mathrm{C}}$ \\
\hline \multicolumn{8}{|l|}{ Histologic diagnosis } \\
\hline DFSP & $54(56.84)$ & $297.5(45-2,451)$ & $1.000^{d}$ & $260(17-4,260)$ & $0.613^{d}$ & $11(0-3,078)$ & $1.000^{d}$ \\
\hline MFS & $12(12.63)$ & $235.5(17-2,419)$ & $1.000^{\mathrm{e}}$ & $392.5(11-4,487)$ & $0.638^{e}$ & $43(0-1,582)$ & $1.000^{\mathrm{e}}$ \\
\hline ATFS & $29(30.53)$ & $93(13-3,753)$ & $1.000^{f}$ & $208(28-4,517)$ & $1.000^{f}$ & $18(0-1,997)$ & $1.000^{f}$ \\
\hline \multicolumn{8}{|l|}{ TNM staging } \\
\hline T1aNOMO+T1bNOMO & $63(66.32)$ & $228(13-3,753)$ & 0.139 & $224(11-4,517)$ & 0.094 & $11(0-1,997)$ & 0.120 \\
\hline T2aNOMO+T2bNOMO & 32 (33.68) & $274.5(22-2,451)$ & & $322(51-4,487)$ & & $32(0-3,078)$ & \\
\hline \multicolumn{8}{|l|}{ Tumor grade } \\
\hline G1 & $55(57.89)$ & $242(17-2,102)$ & $0.730^{9}$ & $237(11-4,260)$ & $0.241^{g}$ & $11(0-3,078)$ & $1.000^{\mathrm{g}}$ \\
\hline G2 & $35(36.84)$ & $246(22-3,753)$ & $0.953^{h}$ & $320(36-4,517)$ & $0.758^{\mathrm{h}}$ & $23(0-2,645)$ & $1.000^{\mathrm{h}}$ \\
\hline G3 & $5(5.26)$ & $250(13-2,419)$ & $0.846^{i}$ & $232(121-4,487)$ & $0.550^{i}$ & $38(6-1,581)$ & $0.659^{i}$ \\
\hline \multicolumn{8}{|l|}{ AJCC staging } \\
\hline $\mathrm{IA}+\mathrm{IB}$ & $55(57.89)$ & $242(17-2,102)$ & 0.250 & $237(11-4,260)$ & 0.038 & $11(0-3,078)$ & 0.241 \\
\hline$\|A+\| B+\|\|$ & $40(42.11)$ & $248(13-3,753)$ & & $315.5(36-4,517)$ & & $23.5(0-2,645)$ & \\
\hline
\end{tabular}

a, Skin group vs Extremity group; b, Extremity group vs Trunk and neck group; c, Trunk and neck group vs Skin group; d, DFSP group vs MFS group; e, MFS group vs ATFS group; $f$, ATFS group vs DFSP group; g, G1 vs G2; h, G2 vs G3; i, G3 vs G1; using one-way ANOVA followed by Bonferroni's test or by Dunnett's T3 test if the requirements of normal distribution and equal variance were not fulfilled.

Abbreviations: N, Number; TNM, Tumor Node Metastasis; AJCC, American Joint Committee on Cancer; DFSP, Dermatofibrosarcoma protuberans; MFS, Myxofibrosarcoma; ATFS, Adult-type fibrosarcoma; $C D$, cluster of differentiation.

The relationship between HMGB1 and tumor-related myeloid cells in fibroblastic sarcoma is unclear. In this study, a visible number of TAMs and CD33-positive myeloid cells were observed in most tumor tissues. It is generally accepted that TAMs possess M2polarized macrophages which play an immunosuppressive role and promote tumor progression $[34,35]$. Although there was similar density of CD68 (staining pan-macrophages) and CD163 (staining M2-polarized macrophages) in fibroblastic sarcoma, only high density of CD163 indicated higher tumor staging. This means that M2-polarized macrophages play a more critical role in the progression of fibroblastic sarcoma. Consistent with this, in some tumor types, including head and neck squamous cell carcinoma and esophageal cancer, a higher density of CD163-positive TAMs is closely correlated to worse clinical courses, whereas no significant association is seen between the density of CD68-positive TAMs and clinical prognosis [36, 37]. Abundant accumulation of CD68 and CD163-positive macrophages in sarcoma is also elucidated in a study involving 24 types of sarcoma including DFSP and MFS. The authors also show that the sarcomas tend toward M2-like macrophage polarization [38], further supporting that M2polarized macrophages is involved in sarcomas progression. MDSCs, usually labeled in combination with $\mathrm{CD}_{3} 3^{+}$and other markers, are another important group of tumor-related myeloid cells that accumulate in tumor tissues, promote tumor progression and suppress anti-tumor immune responses [39, 40]. Compared with TAMs, the density of CD33-positive myeloid cells was much lower in fibroblastic sarcoma and not significantly related to disease progression. Higher tumor staging showed a trend of higher density of CD33, which suggests that CD33-positive myeloid cells such as MDSCs also play a part in promoting the development of fibroblastic sarcoma. The correlation between HMGB1 and CD68 has been observed in gastric cancer [41], but the correlation between HMGB1 and CD163 and CD33 has not been reported. In fibroblastic sarcomas, the total score and the cytoplasmstaining score of HMGB1 were positively correlated with CD68, CD163 and CD33 density, indicating that HMGB1 may act on M2-polarized TAMs and CD33-positive myeloid cells in fibroblastic sarcomas and contribute to disease progression. There appears to be a mechanism linking HMGB1 to immunosuppression and myeloid cells including TAMs and CD33-positive myeloid cells in patients with malignant tumors, which needs further studies to uncover.

The present study lacked a survival analysis because follow-up information from the patients was unavailable. Analysis of data from The Cancer Genome Atlas (TCGA) and GSE72545 datasets found that MFS patients with higher transcriptional levels of HMGB1 had a poor prognosis. However, CD68, CD163 and CD33 showed no prognostic value for MFS patients (data not shown). In addition, data on DFSP and ATFS in public databases are very limited, and the relationship between the expression of HMGB1, CD68, CD163 and CD33 and the prognosis of DFSP and ATFS remains further research. 

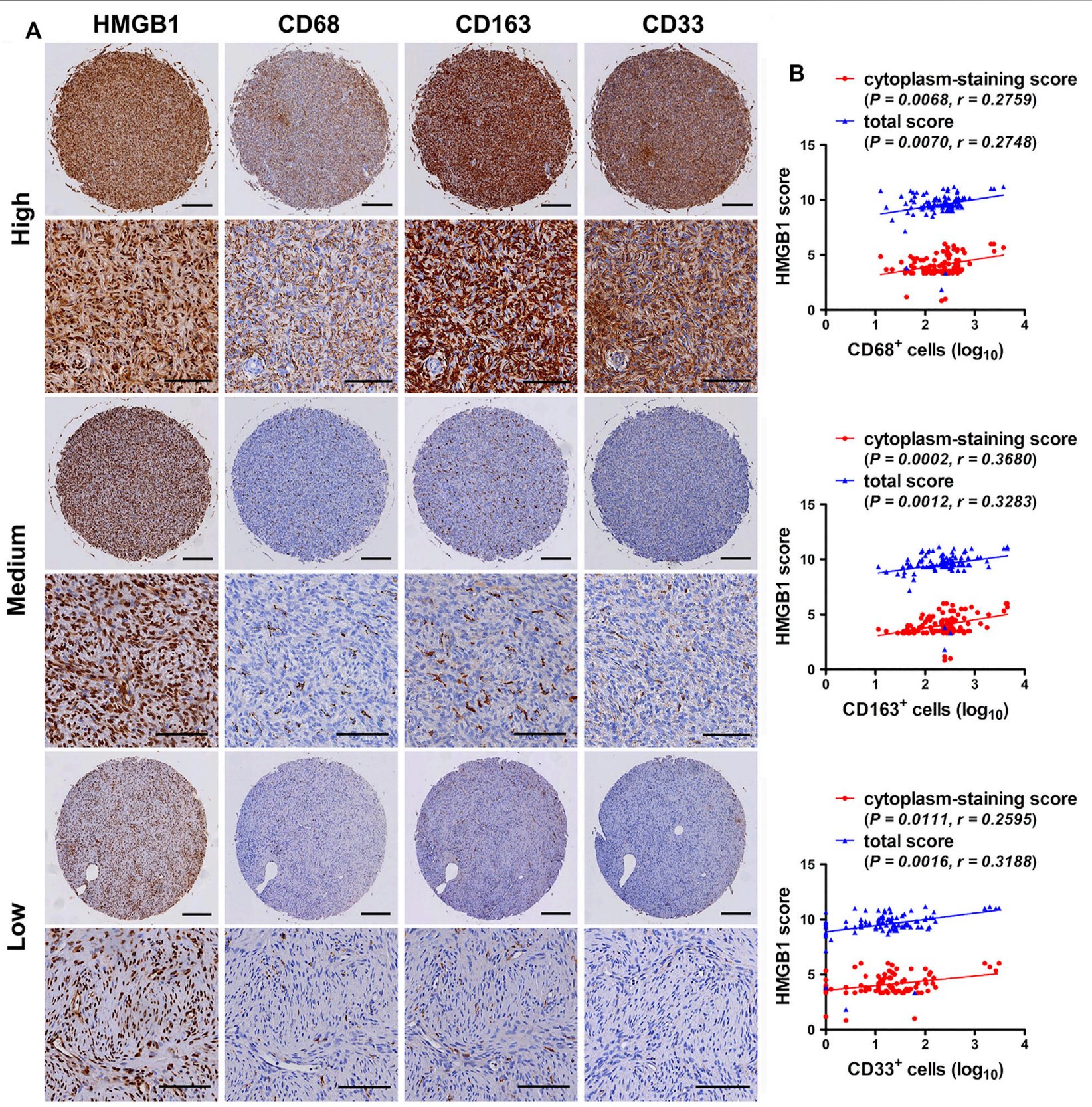

FIGURE 2 | Expression correlation of HMGB1 with CD68, CD163 and CD33 in fibroblastic sarcomas. (A) Immunohistochemical staining for HMGB1, CD68, CD163 and CD33-positive cells in fibroblastic sarcomas. Representative images of high, medium or low staining of all Immunohistochemical markers in one same tissue microarray (TMA) core are shown. Scale bars were $200 \mu \mathrm{m}$ for low magnification ( $\times 4$ ) and $100 \mu \mathrm{m}$ for high magnification ( $\times 200)$. (B) Correlation of HMGB1 total score and cytoplasm-staining score with CD68, CD163 and CD33 density in fibroblastic sarcomas. Cell density was transformed to log10 scale for statistical analysis and plotting. The correlations were analyzed by Spearman rank correlation test.

Another limitation of this study is that no genetic data were provided on the possible cause of HMGB1 overexpression in fibroblastic sarcomas. By using the cbioportal (http://www.cbioportal.org/), a powerful online website for integrated cancer data analysis, the relationship between HMGB1 mRNA levels and gene mutations, copynumber alterations and methylation in MFS cases from TCGA was analyzed. The results showed that no HMGB1 mutations were detected in patients with MFS. Higher mRNA level of HMGB1 was found in gene gain and diploid group. Moreover, the mRNA level of HMGB1 was significantly negatively correlated with the level of methylation; indicating that high expression of HMGB1 in MFS is related to gene amplification and hypomethylation (data not shown). Similarly, whether this scenario occurs in DFSP and ATFS remains further investigation. 
In summary, this study implies that HMGB1 released by tumor cells and vascular endothelial cells may modulate the infiltration, polarization and function of TAMs and CD33positive myeloid cells, thereby contributing to the progression of fibroblastic sarcomas. Although these conclusions require further confirmation via in vivo and in vitro experiments, this study provides clues furthering our understanding of the role of HMGB1 in the progression of fibroblastic sarcomas, and suggests that HMGB1 may serve as a useful prognostic biomarker and a potential therapeutic target for the disease.

\section{DATA AVAILABILITY STATEMENT}

The original contributions presented in the study are included in the article/Supplementary Material, further inquiries can be directed to the corresponding author/s.

\section{ETHICS STATEMENT}

The studies involving human participants were reviewed and approved by the Ethics Committee of People's Hospital of Tongxu County, Henan Province, China. No informed consent is necessary in the scope of this study, since the specimen were analyzed retrospectively and already belonged to National Human Genetic Resources Sharing Service Platform (Library number: 2005DKA21300) at the time of the analyses.

\section{REFERENCES}

1. Cancer Research UK. Soft tissue sarcoma statistics (2013): Available from: https://www.cancerresearchuk.org/health-professional/cancer-statistics/ statistics-by-cancer-type/soft-tissue-sarcoma (Accessed June 1st, 2020).

2. Coffin CM, Alaggio R. Fibroblastic and myofibroblastic tumors in children and adolescents. Pediatr Dev Pathol (2012) 15(1 Suppl. 1):127-80. doi:10.2350/1012-0944-PB.1

3. CDM Fletcher, JA Bridge, P Hogendoorn, F Mertens, editors. World Health Organization classification of tumours of soft tissue and bone. 4th ed. LyonBordeaux, France: IARC Press (2013).

4. Meyer M, Seetharam M. First-line therapy for metastatic soft tissue sarcoma. Curr Treat Options Oncol (2019) 20(1):6. doi:10.1007/s11864-019-0606-9

5. Sabolch A, Feng M, Griffith K, Rzasa C, Gadzala L, Feng F, et al. Risk factors for local recurrence and metastasis in soft tissue sarcomas of the extremity. Am J Clin Oncol (2012) 35(2):151-7. doi:10.1097/COC.0b013e318209cd72

6. Chawla SP, Papai Z, Mukhametshina G, Sankhala K, Vasylyev L, Fedenko A, et al. First-line aldoxorubicin vs doxorubicin in metastatic or locally advanced unresectable soft-tissue sarcoma. JAMA Oncol (2015) 1(9):1272-80. doi:10. 1001/jamaoncol.2015.3101

7. Gupta S, Gouw L, Wright J, Chawla S, Pitt D, Wade M, et al. Phase II study of amrubicin (SM-5887), a synthetic 9-aminoanthracycline, as first line treatment in patients with metastatic or unresectable soft tissue sarcoma: durable response in myxoid liposarcoma with TLS-CHOP translocation. Invest New Drugs (2016) 34(2):243-52. doi:10.1007/s10637-016-0333-z

8. Schöffski P, Chawla S, Maki RG, Italiano A, Gelderblom H, Choy E, et al. Eribulin versus dacarbazine in previously treated patients with advanced liposarcoma or leiomyosarcoma: a randomised, open-label, multicentre, phase 3 trial. The Lancet (2016) 387(10028):1629-37. doi:10.1016/S0140-6736(15)01283-0

\section{AUTHOR CONTRIBUTIONS}

Conception and design: HC, SX, JW; Development of methodology: HC, HL, JA, SX; Acquisition of data: HC, XL, RL, JA; Histological and immunophenotypical revision and diagnosis confirmation: SX; Analysis and interpretation of data (e.g., statistical analysis, computational analysis): $\mathrm{HC}, \mathrm{XL}, \mathrm{CH}$; SX; Writing, review, and/or revision of the manuscript: HC, XL, SX, JW; Study supervision: SX, JW. All authors have seen and approved the final version of the manuscript being submitted.

\section{FUNDING}

This work was supported by the National Natural Science Foundation of China (Grant No. 81860474); Natural Science Foundation of Guangxi Zhuang Autonomous Region (Grant No. 2018GXNSFBA281036); Science and Technology Plan Project of Guangxi Zhuang Autonomous Region (Grant No. GuiKeAD19110011); and The Ph.D. Programs Foundation of Guangdong Second Provincial General Hospital (Grant No. 2019BSGZ016).

\section{CONFLICT OF INTEREST}

The authors declare that the research was conducted in the absence of any commercial or financial relationships that could be construed as a potential conflict of interest.

9. Merchant MS, Wright M, Baird K, Wexler LH, Rodriguez-Galindo C, Bernstein $\mathrm{D}$, et al. Phase I clinical trial of ipilimumab in pediatric patients with advanced solid tumors. Clin Cancer Res (2016) 22(6):1364-70. doi:10.1158/1078-0432. CCR-15-0491

10. Tawbi HA, Burgess M, Bolejack V, Van Tine BA, Schuetze SM, Hu J, et al. Pembrolizumab in advanced soft-tissue sarcoma and bone sarcoma (SARC028): a multicentre, two-cohort, single-arm, open-label, phase 2 trial. Lancet Oncol (2017) 18(11):1493-501. doi:10.1016/S1470-2045(17)30624-1

11. Campana L, Bosurgi L, Rovere-Querini P. HMGB1: a two-headed signal regulating tumor progression and immunity. Curr Opin Immunol (2008) 20(5):518-23. doi:10.1016/j.coi.2008.04.012

12. Kang R, Zhang Q, Zeh HJ, 3rd, Lotze MT, Tang D. HMGB1 in cancer: good, bad, or both? Clin Cancer Res (2013) 19(15):4046-57. doi:10.1158/1078-0432. CCR-13-0495

13. Dajon M, Iribarren K, Cremer I. Toll-like receptor stimulation in cancer: a proand anti-tumor double-edged sword. Immunobiology (2017) 222(1):89-100. doi:10.1016/j.imbio.2016.06.009

14. Sims GP, Rowe DC, Rietdijk ST, Herbst R, Coyle AJ. HMGB1 and RAGE in inflammation and cancer. Annu Rev Immunol (2010) 28:367-88. doi:10.1146/ annurev.immunol.021908.132603

15. Chiba S, Baghdadi M, Akiba H, Yoshiyama H, Kinoshita I, Dosaka-Akita H, et al. Tumor-infiltrating DCs suppress nucleic acid-mediated innate immune responses through interactions between the receptor TIM-3 and the alarmin HMGB1. Nat Immunol (2012) 13(9):832-42. doi:10.1038/ni.2376

16. Xu J, Li J, Yu Z, Rao H, Wang S, Lan H. HMGB1 promotes HLF-1 proliferation and ECM production through activating HIF1- $\alpha$-regulated aerobic glycolysis. Pulm Pharmacol Ther (2017) 45:136-41. doi:10.1016/j.pupt.2017.05.015

17. Park SY, Lee SW, Kim HY, Lee WS, Hong KW, Kim CD. HMGB1 induces angiogenesis in rheumatoid arthritis via HIF-1a activation. Eur J Immunol (2015) 45(4):1216-27. doi:10.1002/eji.201444908 
18. Di Maggio S, Milano G, De Marchis F, D’Ambrosio A, Bertolotti M, Palacios BS, et al. Non-oxidizable HMGB1 induces cardiac fibroblasts migration via CXCR4 in a CXCL12-independent manner and worsens tissue remodeling after myocardial infarction. Biochim Biophys Acta (Bba) Mol Basis Dis (2017) 1863(11):2693-704. doi:10.1016/j.bbadis.2017.07.012

19. Takeuchi A, Yamamoto Y, Munesue S, Harashima A, Watanabe T, Yonekura H, et al. Low molecular weight heparin suppresses receptor for advanced glycation end products-mediated expression of malignant phenotype in human fibrosarcoma cells. Cancer Sci (2013) 104(6):740-9. doi:10.1111/cas.12133

20. Apetoh L, Ghiringhelli F, Tesniere A, Obeid M, Ortiz C, Criollo A, et al. Tolllike receptor 4-dependent contribution of the immune system to anticancer chemotherapy and radiotherapy. Nat Med (2007) 13(9):1050-9. doi:10.1038/ nm1622

21. Gulic T, Silva-Gomes R, Davoudian S, Sironi M, Allavena P, Mantovani A, et al. Tumor-associated myeloid cells in cancer progression. In: N Rezaei, editor. Cancer immunology. Second edn. Cham, Switzerland: Springer (2020). p. 29-46. doi:10.1007/978-3-030-30845-2_3

22. Evankovich J, Cho SW, Zhang R, Cardinal J, Dhupar R, Zhang L, et al. High mobility group box 1 release from hepatocytes during ischemia and reperfusion injury is mediated by decreased histone deacetylase activity. J Biol Chem (2010) 285(51):39888-97. doi:10.1074/jbc.M110.128348

23. Bronte V, Brandau S, Chen S-H, Colombo MP, Frey AB, Greten TF, et al. Recommendations for myeloid-derived suppressor cell nomenclature and characterization standards. Nat Commun (2016) 7:12150. doi:10.1038/ ncomms 12150

24. Taguchi A, Blood DC, del Toro G, Canet A, Lee DC, Qu W, et al. Blockade of RAGE-amphoterin signalling suppresses tumour growth and metastases. Nature (2000) 405(6784):354-60. doi:10.1038/35012626

25. Liu L, Yang M, Kang R, Wang Z, Zhao Y, Yu Y, et al. HMGB1-induced autophagy promotes chemotherapy resistance in leukemia cells. Leukemia (2011) 25(1):23-31. doi:10.1038/leu.2010.225

26. Liang X, Romo de Vivar Chavez A, Schapiro NE, Loughran P, Thorne SH, Amoscato AA, et al. Ethyl pyruvate administration inhibits hepatic tumor growth. J Leukoc Biol (2009) 86(3):599-607. doi:10.1189/jlb.0908578

27. Tang D, Kang R, Cheh C-W, Livesey KM, Liang X, Schapiro NE, et al. HMGB1 release and redox regulates autophagy and apoptosis in cancer cells. Oncogene (2010) 29(38):5299-310. doi:10.1038/onc.2010.261

28. Ohndorf U-M, Rould MA, He Q, Pabo CO, Lippard SJ. Basis for recognition of cisplatin-modified DNA by high-mobility-group proteins. Nature (1999) 399(6737):708-12. doi:10.1038/21460

29. Suren D, Arda Gokay A, Sayiner A. High mobility group box 1 (HMGB1) expression in gastric adenocarcinomas. J BUON (2018) 23(2):422-7.

30. Dong Y-D, Cui L, Peng C-H, Cheng D-F, Han B-S, Huang F. Expression and clinical significance of HMGB1 in human liver cancer: knockdown inhibits tumor growth and metastasis in vitro and in vivo. Oncol Rep (2013) 29(1): 87-94. doi:10.3892/or.2012.2070
31. Lee HJ, Kim A, Song IH, Park IA, Yu JH, Ahn JH, et al. Cytoplasmic expression of high mobility group B1 (HMGB1) is associated with tumor-infiltrating lymphocytes (TILs) in breast cancer. Pathol Int (2016) 66(4):202-9. doi:10.1111/pin.12393

32. Takeuchi T, Sakazume K, Tonooka A, Zaitsu M, Takeshima Y, Mikami K, et al. Cytosolic HMGB1 expression in human renal clear cell cancer indicates higher pathological T classifications and tumor grades. Urol J (2013) 10(3):960-5. doi:10.22037/uj.v10i3.1425

33. Lee HJ, Kim JY, Song IH, Park IA, Yu JH, Ahn J-H, et al. High mobility group $\mathrm{B} 1$ and N1 (HMGB1 and HMGN1) are associated with tumor-infiltrating lymphocytes in HER2-positive breast cancers. Virchows Arch (2015) 467(6): 701-9. doi:10.1007/s00428-015-1861-1

34. Chanmee T, Ontong P, Konno K, Itano N. Tumor-associated macrophages as major players in the tumor microenvironment. Cancers (2014) 6(3):1670-90. doi:10.3390/cancers6031670

35. Komohara Y, Fujiwara Y, Ohnishi K, Takeya M. Tumor-associated macrophages: potential therapeutic targets for anti-cancer therapy. $A d v$ Drug Deliv Rev (2016) 99(Pt B):180-5. doi:10.1016/j.addr.2015.11.009

36. Troiano G, Caponio VCA, Adipietro I, Tepedino M, Santoro R, Laino L, et al. Prognostic significance of CD68+ and CD163+ tumor associated macrophages in head and neck squamous cell carcinoma: a systematic review and meta-analysis. Oral Oncol (2019) 93:66-75. doi:10.1016/j. oraloncology.2019.04.019

37. Li J, Xie Y, Wang X, Li F, Li S, Li M, et al. Prognostic impact of tumorassociated macrophage infiltration in esophageal cancer: a meta-analysis. Future Oncol (2019) 15(19):2303-17. doi:10.2217/fon-2018-0669

38. Dancsok AR, Gao D, Lee AF, Steigen SE, Blay J-Y, Thomas DM, et al. Tumorassociated macrophages and macrophage-related immune checkpoint expression in sarcomas. Oncoimmunology (2020) 9(1):1747340. doi:10.1080/ 2162402X.2020.1747340

39. Ostrand-Rosenberg S, Fenselau C. Myeloid-derived suppressor cells: immunesuppressive cells that impair antitumor immunity and are sculpted by their environment. J.I. (2018) 200(2):422-31. doi:10.4049/jimmunol.1701019

40. Tcyganov E, Mastio J, Chen E, Gabrilovich DI. Plasticity of myeloid-derived suppressor cells in cancer. Curr Opin Immunol (2018) 51:76-82. doi:10.1016/j. coi.2018.03.009

41. Akaike H, Kono K, Sugai H, Takahashi A, Mimura K, Kawaguchi Y, et al. Expression of high mobility group box chromosomal protein-1 (HMGB-1) in gastric cancer. Anticancer Res (2007) 27(1A):449-57.

Copyright (c) 2021 Chen, Lin, Liu, Huang, Li, Ai, Wei and Xiao. This is an openaccess article distributed under the terms of the Creative Commons Attribution License (CC BY). The use, distribution or reproduction in other forums is permitted, provided the original author(s) and the copyright owner(s) are credited and that the original publication in this journal is cited, in accordance with accepted academic practice. No use, distribution or reproduction is permitted which does not comply with these terms. 